\title{
Remedial effects of motivational incentive on declining cognitive control in healthy aging and Parkinson's disease
}

\author{
Helga A. Harsay ${ }^{1+}$, Jessika I. V. Buitenweg ${ }^{1+}$, Jasper G. Wijnen' , Maria J. S. Guerreiro ${ }^{2}$ and K. Richard \\ Ridderinkhof ${ }^{1,3 *}$
}

Department of Psychology, Amsterdam Center for the Study of Adaptive Control in Brain and Behavior (Acacia), University of Amsterdam, Amsterdam, Netherlands
2 Department of Neuropsychology and Psychopharmacology, University of Maastricht, Maastricht, Netherlands
${ }_{3}$ Cognitive Science Center Amsterdam, University of Amsterdam, Amsterdam, Netherlands

\section{Edited by:}

Lars Nyberg, Umeå University,

Sweden

Reviewed by:

Lars Forsgren, Umeå University,

Sweden

Petter Marklund, Stockholm University,

Sweden

*Correspondence:

K. Richard Ridderinkhof, Department of Psychology, University of Amsterdam

Roetersstraat 15, 1018 WB

Amsterdam, Netherlands.

e-mail:k.r.ridderinkhof@uva.nI

${ }^{+}$Helga A. Harsay and Jessika I. V.

Buitenweg have contributed equally to this work.
The prospect of reward may provide a motivational incentive for optimizing goal-directed behavior. Animal work demonstrates that reward-processing networks and oculomotor-control networks in the brain are connected through the dorsal striatum, and that reward anticipation can improve oculomotor control via this nexus. Due perhaps to deterioration in dopaminergic striatal circuitry, goal-directed oculomotor control is subject to decline in healthy seniors, and even more in individuals with Parkinson's disease (PD). Here we examine whether healthy seniors and PD patients are able to utilize reward prospects to improve their impaired antisaccade performance. Results confirmed that oculomotor control declined in PD patients compared to healthy seniors, and in healthy seniors compared to young adults. However, the motivational incentive of reward expectation resulted in benefits in antisaccade performance in all groups alike. These findings speak against structural and non-modifiable decline in cognitive control functions, and emphasize the remedial potential of motivational incentive mechanisms in healthy as well as pathological aging.

Keywords: healthy aging, Parkinson's disease, cognitive control, decline, optimization, reward anticipation, action preparation, antisaccade

\section{INTRODUCTION}

Healthy elderly adults and patients with Parkinson's disease (PD) are generally less proficient in the cognitive control of goaldirected actions than healthy young adults. Proficiency in cognitive control involves a wide range of processes that are relevant for personal independence in everyday task performance. A key characteristic is the cognitive anticipation of actions that need to be executed. This capacity is affected by aging (Falkenstein et al., 2006; Roggeveen et al., 2007; Sterr and Dean, 2008) and in Parkinson patients. Impaired cognitive anticipation has been observed in several cognitive control tasks such as the antisaccade task and in task switching tasks. In the Stroop task, as well as in oculomotor switch tasks, switch costs increase with PD, specifically when patients switch from a more automatic to a more voluntary response (Woodward et al., 2002).

Some generic factors have been found to alleviate these problems in the elderly. Performance improves, for instance, if specific information on the upcoming response is available in advance (Sterr and Dean, 2008). Likewise, older adults may benefit from prolonged preparation time (Loveless and Sanford, 1974; BoninGuillaume et al., 2000).

In the current study, we are interested in whether action preparation can be optimized using motivational factors. One powerful motivator of goal-directed behavior is the prospect of obtaining a reward. Rewards come in many shapes and forms: apples, juices, money, and even symbolic tokens (e.g., game points) are considered powerful rewards. The weighting of the potential rewards and punishments associated with behavioral options is instrumental to efficient behavior (Opris and Bruce, 2005; Schultz, 2006). Expectations of upcoming rewards influence systems concerned with action preparation and motor control. Indeed, for primates (Lauwereyns et al., 2002; Kawagoe et al., 2004) as well as humans (Ramnani and Miall, 2003), it is shown that actions are executed with greater efficiency when their goals are rewarded. Reward thus seems to play an important role in shaping behavior, but it is unclear as to whether it can also exert remedial effects on behaviors that show signs of decline, such as those associated with aging. Given that the neural substrates of reward processing are deemed to be vulnerable to the effects of advancing age (Marschner et al., 2005; Mell et al., 2005, 2009; Schott et al., 2007; Weiler et al., 2008), remedial effects may in fact be unobservable.

To investigate the remedial potential of reward expectations on declining action preparation, we focus on antisaccade performance. Antisaccades are particularly demanding in terms of action preparation (Reuter et al., 2006), and the efficiency of this type of process declines with age and in PD. For the generation of antisaccades, subjects are to inhibit an eye movement toward a peripheral stimulus and instead generate an eye movement in the opposite direction (Hallett, 1978; Munoz and Everling, 2004). This requires deliberate control over automatic reflexes. The onset latency of the antisaccade indexes the level of preparation; the shorter the latency, the better one is prepared (Milstein and Dorris, 2007). Latency may be more informative as to the level of preparation than accuracy; elderly tend to display low error rates as they prefer accuracy over speed, even when forced to react fast (Smith and Brewer, 1995). PD patients show increased onset latencies compared to healthy 
elderly, and elderly in turn display increased antisaccade onset latencies when compared to young adults, reflecting progressive decline in preparation processes. PD patients also display difficulty suppressing reflexive prosaccades and premature responses, reflecting patients' greater difficulty with self-initiation of movement than with performing externally guided movement (Butler et al., 1999; Klein et al., 2000; Nieuwenhuis et al., 2000; Chan et al., 2005; Amador et al., 2006). Antisaccades engage multiple cognitive processes including working memory, response inhibition, incompatible stimulus-response mapping and action preparation. Many studies have discussed age-related decrements in this task with regard to the involvement of inhibitory control (Butler et al., 1999; Nieuwenhuis et al., 2000; Sweeney et al., 2001; Butler and Zacks, 2006), working memory (Eenshuistra et al., 2004), and goal neglect (Nieuwenhuis et al., 2004).

The conditions under which age-related and PD-related antisaccadic decline can be improved, a topic of particular relevance for the current study, have been addressed less extensively. Little is known about the influence of reward expectation on age-related and pathology-related decline of oculomotor behavior. Oculomotor tasks have been shown to be sensitive to reward manipulations in non-human primates (Lauwereyns et al., 2002; Hikosaka et al., 2006), in healthy young adults (Milstein and Dorris, 2007), and in depressed and anxious individuals (Jazbec et al., 2005). The interface between motivation and declining action is central to the current behavioral study. In this study our focus will be on the influence of reward anticipation on declining oculomotor preparation.

To examine effects of reward anticipation on oculomotor preparation in elderly and $\mathrm{PD}$ patients we used the antisaccade paradigm. We adopted a variation of Rosenbaum's (1980) precuing technique where different amounts and types of advance information were provided to the participants. Motivational instruction cues informed the participant in advance that a reward is at stake when the antisaccade action is performed well. Preparatory instruction provided specific spatial information on the upcoming response, e.g., the side of the screen where the peripheral cue, that indexes the direction of the antisaccade response, will appear. This paradigm builds on existing frameworks of oculomotor control non-human primates linking similar corticostriatal mechanisms of reward anticipation with those of action preparation (Sato and Hikosaka, 2002; Hikosaka et al., 2006; Nakamura and Hikosaka, 2006) and explores their potential for remediating declining cognitive control in aging and PD.

While healthy aging involves a certain degree of dopaminergic cell loss in the basal ganglia, specifically in the substantia nigra pars compacta (SNc) (Hubble, 1998; Marschner et al., 2005) and bilateral shrinkage of caudate and putamen (Gunning-Dixon et al., 1998), PD is commonly associated with a more profound degeneration (e.g., more than $70 \%$ of dopaminergic cells in the $\mathrm{SNc}$ ). The age-related loss of SNc cells doesn't seem to constitute an important factor in the pathogenesis of $\mathrm{PD}$, as other parts of the SNc degenerate in aging than in PD. PD dominantly affects the lateral and medial ventral tier of the SNc (average loss 91\%), a part that is relatively spared in aging $(2.1 \%$ loss per decade) (Fearnley and Lees, 1991). Direct correlations have been reported between measures of PD related dopaminergic denervation in the caudate and cognitive dysfunction (Cropley et al., 2006) such as impaired verbal episodic memory (Holthoff-Detto et al., 1997), attention and response inhibition (Rinne et al., 2000; Bruck et al., 2005). These findings are consistent with reports that executive dysfunction in PD is accompanied by reduced activity within the caudate nucleus (Marklund et al., 2009) and prefrontal regions (Lewis et al., 2003). Marklund et al. (2009) observed a decrease in caudate activation that was transient rather than sustained in nature, which may suggest that the differential caudate activation stemmed from PD-related deficiencies in phasic dopamine release.

These neural changes in caudate nucleus also affect the neural underpinnings of antisaccades because antisaccade action requires cortico-subcortical integration. The widespread oculomotor network encompasses, besides the eye fields in all the major cortices and portions of the visual cortex, several structures within the basal ganglia (Raemaekers et al., 2006). The basal ganglia component of the oculomotor network originates in the caudate and then converges on the substantia nigra reticulata $(\mathrm{SNr})$, which in turn projects to the oculomotor-executor in the brainstem, the superior colliculus, to modulate saccadic eye-movement. Direct projections between caudate and superior colliculus have also been observed. The superior colliculus receives excitatory inputs from many cortical brain areas, when stimuli attract attention and gaze. These inputs are, however, often incapable of activating superior colliculus neurons, because the $\mathrm{SNr}$ (with the help of the caudate) acts as a gate for saccade generation by inducing GABAergic inhibition on the superior colliculus. Only when the caudate/SNr reduce inhibition, do the superior colliculus neurons spike leading to action execution (Hikosaka et al., 2000). Altered patterns of SNr firing such as single-spike or burst firing are found in Parkinson's disease (Tseng et al., 2000). The underlying decrements of these altered patterns in the $\mathrm{SNr}$ are not clear. They presumably stem from decrements in the dopamine release of the ventral projecting dendrites from the SNc to the SNr (Prescott et al., 2009).

Taken together, the progressive decline in antisaccade performance in aging and PD may be associated with progressive dopaminergic cell loss in the $\mathrm{SNc}$ to the caudate/SNr inflicting a reduction of the caudate/SNr inhibition on the superior colliculus. Antisaccadic deficits are also observed in other basal ganglia disorders, including Huntington's disease. The similarity between Parkinson's and Huntington's diseases is noteworthy because they are caused by different mechanisms, the former by a loss of neurons mainly in the SNc and the latter by a loss of neurons mainly in the caudate. This suggests that also in humans, the SN and the caudate work together for the control of saccadic eye movements (Hikosaka et al., 2006). Accordingly, the antisaccade task has been found to be sensitive to dopamine changes; in PD patients the number of errors on the antisaccade task decreases with levodopa medication (Hood et al., 2007).

Importantly, the caudate is not only involved in anticipatory antisaccade programming, but also in anticipatory motivational processes within the mesolimbic dopamine system (Hikosaka et al., 2006). Many caudate neurons fire not only before the onset of an expected saccadic target but also in preparatory responding to signals that predict reward. Reward-related processes are prevalent in the entire striatum (caudate, putamen, nucleus accumbens), which receives massive input from the limbic system (e.g., amygdala, orbitofrontal cortex Selemon and Goldman-Rakic, 1985; Fudge et al., 
2002). Functionally, the caudate specifically is thought to reinforce plans for complex behavior (Kawagoe et al., 1998; Cromwell and Schultz, 2003; O'Doherty et al., 2004) based on an evaluation of action-outcomes provided by the nucleus accumbens, who is less selective for sensorimotor events (Schultz et al., 1992). The caudate thus plays a key role in sensorimotor/cognitive action preparation on the basis of motivation.

Consistently, a number of primate studies have underlined the crucial role of the caudate nucleus in transforming motivational information into eye movement signals. In an oculomotor task, neurons of the primate caudate nucleus were found to respond in anticipation of reward-predicting stimuli, which in turn modulated neural oculomotor signals (Kawagoe et al., 1998, 2004) and corresponded to the saccade onset latency (Takikawa et al., 2002). This mechanism of motivational control of saccadic eye movement in caudate nucleus is supported by initial human findings, where participants received monetary rewards, following an anticipatory cue or following a button press response (Tricomi et al., 2004). Results showed only differential activation in the caudate nucleus if a perception of contingency existed between a button press response and a reward outcome.

Such a nexus between the oculomotor- and reward systems may be instrumental for the optimization of antisaccade performance in seniors and PD patients. In PD, the most rostro-dorsal part of the caudate is subjected to greater disruption than the relatively spared ventral region of the caudate (Grahn et al., 2008). This may leave the ventral connections of the caudate relatively intact and receptive for input from the ventral striatum and the limbic nodes of the reward circuit.

Thus oculomotor preparation may be facilitated by a transient increase of caudate activation as induced by the prospect of reward (Lauwereyns et al., 2002; Watanabe and Hikosaka, 2005), and this facilitation might alleviate the deficits associated with aging and PD. However, reward processing in itself is also affected by age and PD. Older adults have shown greater difficulty in learning reward associations, needing more trials before reaching the learning criterion, compared to young adults (Mell et al., 2005). Similar behavioral decrements have been found in non-medicated PD patients (Bodi et al., 2009).PD patients show poorer performance on reward-motivated probabilistic classification learning. These decrements have been related to changes in anticipatory reward cue processes. Neural evidence on age- and PD-related changes in the reward system is not unequivocal. While Samanez-Larkin et al. (2007) observed intact ventral striatal activation (and activation of the medial caudate and anterior insula) during gain anticipation in both younger and older adults, Schott et al. (2007) observed ventral striatal activation during reward anticipation only in the young. Healthy elderly and PD patients did not activate the ventral striatum during reward anticipation, only during reward feedback. As compared to healthy elderly, PD patients showed additional functional alterations in reward cue processing and reduced functional connectivity between the nucleus accumbens and the ventral tegmental area. Neural decrements in reward cue processing presumably reflect pre-synaptic degeneration of dopamine neurotransmission in $\mathrm{PD}$ and (milder) changes during aging in both the pre-and postsynaptic dopamine system throughout the human brain (Kaasinen et al., 2000; Bäckmann and Farde, 2001; Backman et al., 2006). This age-related vulnerability of the reward system has been further taken to explain loss of cognitive flexibility in older ages, by leading to impairments in reward processing, stimulus-response association learning and adaptation of existing associations to new situations (Marschner et al., 2005; Mell et al., 2005, 2009; Weiler et al., 2008). On the other hand, PD patients demonstrate increased search efficiency with increasing reward (Goerendt et al., 2004). Also, when required to adapt their manual force to an increasing reward, patients were able to exhibit greater force on trials in which larger rewards can be won, showing the same proportional increase as in controls (Schmidt et al., 2008).

Reward processing thus appears to be affected by age and PD in some studies but not in others. In this state of affairs the question whether deficient oculomotor preparation among seniors and PD patients can be facilitated by the prospect of reward remains unanswered. The presumably more intact ventral and limbic reward structures might trigger a transient increase of caudate activation, thereby enhancing inhibitory forces on the superior colliculus to improve goal-directed oculomotor function. The mesolimbic dopamine system may thus play a compensatory role in "boosting" the efficiency of interactions between motivational and impaired cognitive control processes in healthy aging and PD.

To address this question, we administered an antisaccade task to healthy young and older adults and PD patients, providing them pseudorandomly with the prospect of reward on some trials but not on others. This pseudorandom reward cuing is based on the principle that DA neurons in the reward system respond to the cue positively (with a phasic increase in firing) if the cue indicates an upcoming reward and they respond to the cue negatively (with a phasic decrease in firing) if the cue indicates no reward (Schultz et al., 1992; Kawagoe et al., 2004).

We expected older (compared to young) adults to display deficient oculomotor preparation (as indexed by increased antisaccade latencies), whilst PD patients were expected to perform worse than healthy elderly. We hypothesized further that oculomotor preparation would benefit from the prospect of reward. Most importantly, we examined whether such reward benefits can be observed in healthy elderly and in PD patients. In addition we manipulated spatial preparation and the duration of the interval between the instruction cue and the target, both to replicate typical findings and to ensure that our approach has the potential for oculomotor preparation benefits to become manifest even in older adults and PD patients. Thus we expected antisaccade latencies to be shorter and accuracy to be higher after specific preparation cues providing spatial information on the upcoming response, than after neutral preparation cues that give no spatial information. Likewise, we expected faster and more accurate antisaccades after long compared to shorter cue-target intervals.

\section{MATERIALS AND METHODS PARTICIPANTS}

Eighteen young adults (age 18-36, $M=24.0,9$ female), 18 elderly adults (age 64-85, $M=69.4,9$ female) and $20 \mathrm{PD}$ patients (age $45-79, M=61.8,9$ female) participated in the experiment after giving written informed consent. They were paid an amount of $€ 37$ for participation and 5 cents for every correct reward trial with a maximum of $€ 6.40$ for two blocks. Young adults were students at the University of Amsterdam. Elderly were recruited from a database of healthy elderly subjects ${ }^{1}$ who had previously expressed their

${ }^{1}$ www.SeniorLab.nl 
interest in participation in cognitive aging research. Patients were recruited through Dutch national websites dedicated to PD's disease. Participants had normal or corrected-to normal vision, measured with the Landolt ring chart and were matched on IQ (crystallized IQ as measured by the "Nederlandse Lees Test Volwassenen" (NLV) and fluid IQ as measured by the "Raven Complex Forms"). None of the young and senior participants had a history of neurological or psychiatric disorders, mild cognitive impairment, eye movement or vision problems, or was taking any drugs influencing the central nervous system. The PD patients fulfilled formal diagnostic criteria for PD according to the Unified Parkinson's Disease Rating Scale (UPDRS), had a mean disease duration of 7 years (range of onset 2-13 years) and a mean estimated motor subscore of 16.7 (range 6-38) on the UPDRS which could be labeled as moderately affected when compared to other studies using this measure (Morgante et al., 2006; Harrison et al., 2009). None of the patients suffered from tremors in the neck or head. Patients were asked to continue taking their medication at the required time on the day of testing, and tests were planned 60-90 min after medication intake. Nine patients were receiving dopamine precursors (levodopa/carbidopa), six were taking a monoamine oxidase inhibitor, three were on catechol- $O$-methyl transferase (COMT)-inhibitors and 14 patients were receiving one or more dopamine agonists. One patient did not take medication on a regular basis.

\section{PROCEDURES}

The experiment was divided into a training session and a main experimental session. During training, participants were presented with a series of trials to familiarize themselves with the stimulus-reward associations and antisaccade response requirements. Participants completed a 25-min training block and a 25-min experimental block, each subtending 128 trials, with a 10 -min break between blocks. All experimental procedures were approved by a local ethics committee, and conducted in accordance with the Helsinki Declaration, international laws, and institutional guidelines.

\section{TASK}

\section{Temporal order of stimulus presentation}

Each trial (see Figure 1) started with a central fixation dot surrounded by two square outlines (each subtending $3.8^{\circ}$ visual angle) on the left and right side of the fixation dot (distance $12.4^{\circ}$ ). After the fixation display a central visual instruction cue was presented (for $600 \mathrm{~ms}$ ) followed by a variable cue-target interval of 4.5-6 s, terminated by a peripheral antisaccade target (a white asterisk subtending $2^{\circ}$, displayed for $500 \mathrm{~ms}$ ). The antisaccade target was presented for $500 \mathrm{~ms}$ pseudorandomly in the center of the left or the right square outline. The target indicated that participants should make an immediate eye movement to the opposite side of the screen. Their response was immediately followed by presentation of a feedback image (presented for $500 \mathrm{~ms}$ ).

\section{Feedback: reward, no reward, and error}

The feedback image indicated to participants whether the response was correct or whether the trial was rewarded. On rewarded trials, the reward was symbolically represented as an image of a golden Euro coin. On non-rewarded trials, a silver blank disk of the same size, shape, and luminance was displayed. After an incorrect or too slow response a silver ring with a black circle in the middle was presented. Colors of rewarded, non-rewarded, and error feedback were calibrated to equal luminance using Colorfacts 7 and the color calibration system EyeOneMonitor ${ }^{2}$. Participants were informed that they would receive a monetary reward on golden reward trials in which they performed fast and correctly but not on the silver non-reward trials. In line with some other investigations using monetary reward (Ramnani and Miall, 2003) the exact amount of gain was not displayed in the feedback to avoid mental calculation.

\section{Instruction cues: reward prospect and spatial preparation}

To investigate the effect of reward anticipation and specific preparation on antisaccade performance, we presented instruction cues before the appearance of the peripheral antisaccade target. In a $2 \times 2$ factorial design the instruction cues independently manipulated the level of reward expectation (two levels: reward and no reward expected) and the level of response preparation (two levels: specific preparation or non-specific preparation of the antisaccade response), by means of color and shape.

The level of reward expectation was manipulated by the color of the instruction cue: In reward trials the instruction cue was a gold circle; in no-reward trials the instruction cue

${ }^{2}$ www.datacolor.eu

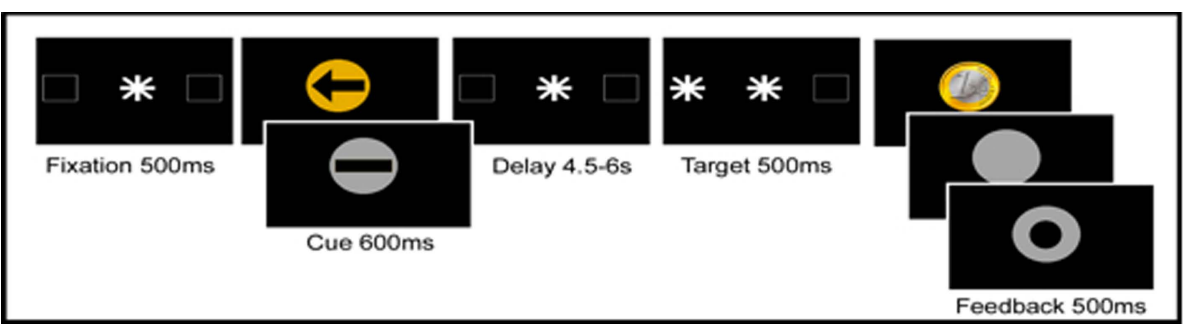

FIGURE 1 |Temporal order of stimuli in the antisaccade task. In reward anticipation trials the instruction cue was a gold circle; in no reward trials the instruction cue was a silver circle. In specific spatial preparation trials, an arrow was displayed, indicating where the subsequent antisaccade target would appear; in neutral non-specific preparation trials, a bar was presented. The length of the preparation interval between the instruction cue and the antisaccade target was varied between 4.5 and $6 \mathrm{~s}$. Immediately after the antisaccade a golden coin was displayed when a reward had been won; a silver circle was presented after a correct response on a non-reward trial, and after an incorrect or slow response a silver ring was displayed. 
was a silver circle. The colors of the reward and the no-reward cue were calibrated to equal luminance using Colorfacts 7 and EyeOneMonitor.

The level of response preparation was manipulated by the content of the instruction cue: in specific preparation trials, an arrow was displayed in the center of the circle, indicating where the subsequent target would appear; in the neutral non-specific preparation trials, a bar replaced the arrow. The arrow enabled subjects to prepare for the appearance of the peripheral antisaccade cue, while a bar would give no information on target location.

\section{EYETRACKING AND STIMULUS DELIVERY SET-UP}

Subjects were seated $60 \mathrm{~cm}$ in front of a computer screen in a dimly lit room with their head stabilized in a chin rest. An infra-red camera was mounted in front of the right pupil in order to monitor eye movements. Eye movements were recorded with ViewPoint Eyetracker PC-60 (Version 2.7, Arrington Research Inc. ${ }^{3}$ ) software on a standard PC. Bidirectional communication between this PC and a second one responsible for the delivery of stimuli (using Presentation software ${ }^{4}$ ) ensured that stimulus onset times were registered in the eye movement data and that adequate feedback was provided to oculomotor responses on each trial. Eye movements were registered with a sampling rate of $60 \mathrm{~Hz}$ along with signals marking the stimulus onset times. Before task onset a 9-point calibration procedure was performed. To eliminate slow drift in eye tracking-signal during the task, calibrated eye position was manually corrected to the central fixation cross. Regions of interest were defined by two peripheral outer square outlines (the endpoints of the antisaccade eye movements) surrounding the central fixation dot. The PC which tracked eye movements, signaled to the stimulus presentation $\mathrm{PC}$ when an eye movement left the fixation region and entered one of the target regions. The Presentation PC recorded correct trials versus errors and presented feedback accordingly.

\section{ANALYSIS}

Saccade parameters were detected with in-house Java-based software ${ }^{5}$ using minimum amplitude $\left(>1.5^{\circ}\right)$ and velocity $\left(>30^{\circ} / \mathrm{s}\right)$ criteria and were subsequently visually inspected and double-checked for accuracy. In line with common definitions (Fischer et al., 1993) saccades with a latency of less than $80 \mathrm{~ms}$ after the display of the peripheral onset target were classified as anticipatory responses. Exclusion criteria applied to trials exceeding $800 \mathrm{~ms}$ (miss), trials with blinks during saccadic execution, trials in which participants failed to focus their eyes on the central cue or in which gaze was not at fixation $200 \mathrm{~ms}$ before target appearance. To avoid latency differences between correct and erroneous antisaccades, incorrect trials were not included in latency analysis. A trial without a premature eye movement toward the peripheral target but with a saccade landing at the location of the square outline on the opposite side of the screen executed within $800 \mathrm{~ms}$ was classified as a correct trial. To investigate premature eye movements, the interval between the preparatory cue and the display of the peripheral antisaccade target was examined for fixation breaks. Fixation breaks were defined as

${ }^{3}$ www.ArringtonResearch.com

${ }^{4}$ www.neurobs.com

${ }^{5}$ www.java.com saccades or antisaccades executed after exposure to the cue and before appearance of the target, with an amplitude at least equal to the mean antisaccade response across trials minus one standard deviation, such that fixation break amplitudes fell within the range of the largest $84.13 \%$ of all saccadic responses (Brown et al., 2007). Antisaccade latencies and accuracy as well as fixation breaks were analyzed using a mixed $3 \times 2 \times 2 \times 2$ ANOVA design with one between-subjects variable (young, elderly, or patient) and three within-subjects variables (reward or no reward; specific- or nonspecific cue; and delay interval of 4500-5000 or 5500-6000 ms). Additional analyses were conducted using a similar ANOVA design, but now with the between-subjects factor consisting of healthy elderly, mild PD patients, and severe PD patients.

\section{RESULTS}

\section{ANTISACCADE LATENCY AND ACCURACY}

As can be seen in Figure 2A, a main effect of Group indicated differences in antisaccade preparation between healthy young adults, healthy elderly, and PD patients $(F(2,53)=65.849, p<0.0001)$. Elderly participants showed a significantly higher antisaccade onset latency compared to young adults $(F(1,34)=9.266, p<0.004)$. PD patients displayed a slight slowing compared to elderly participants $(F(1,36)=1.613, p<0.212)$. As illustrated in Figure 2B, a main effect of Group indicated no differences in correct antisaccade trials between healthy young adults, healthy old adults, and PD patients $(F(2,53)=2.290, p<0.111)$, although $\mathrm{PD}$ patients performed marginally worse than elderly $(F(1,36)=2.932, p<0.095)$.

\section{EFFECT OF REWARD ANTICIPATION ON ANTISACCADE LATENCY AND ACCURACY}

To test for the effect of reward anticipation on antisaccade preparation, we examined the effect of a cue signaling a reward trial as opposed to a cue signaling a no-reward trial on antisaccade latency. This reward benefit effect on latency was highly significant $(F(2,53)=35.855, p<0.0001)$, amounting $31 \mathrm{~ms}$ for young adults $(F(1,17)=13.95, p<0.002), 30 \mathrm{~ms}$ for healthy elderly $(F(1,17)=6.428, p<0.022)$, and $28 \mathrm{~ms}$ for PD patients $(F(1,19)=15.226, p<0.001)$. Importantly, the extent of the improvement was equal in all three groups $(F(2,53)=0.544$, $p<0.583$, see Figure 3A). There was also a highly significant effect of reward anticipation on accuracy $(F(2,53)=26.119, p<0.0001)$, amounting $6.5 \%$ for young adults $(F=12.589, p<0.003), 2,3 \%$ for healthy elderly $(F=5.766, p<0.029)$, and $3.7 \%$ for PD patients $(F=6.568, p<0.020)$. Importantly, no between-group differences in the extent of the improvement were evident between elderly and patients $(3.7 \%)(F=0.723, p<0.401)$ or between patients and young $(F=1.627, p<0.210$, see Figure 3B).

\section{EFFECT OF SPECIFIC PREPARATION ON ANTISACCADE LATENCY AND ACCURACY}

To investigate the effect of specific advance knowledge on action preparation, we examined the effect of a cue providing spatial information as opposed to a neutral cue providing no spatial information. This spatial preparation benefit was highly significant for antisaccade latency $(F(2,53)=65.849, p<0.0001)$, amounting $46 \mathrm{~ms}$ for young adults $(F(1,17)=119.975, p<0.0001), 34 \mathrm{~ms}$ for healthy elderly $(F(1,17)=16.810, p<0.001)$, and $38 \mathrm{~ms}$ for PD patients 

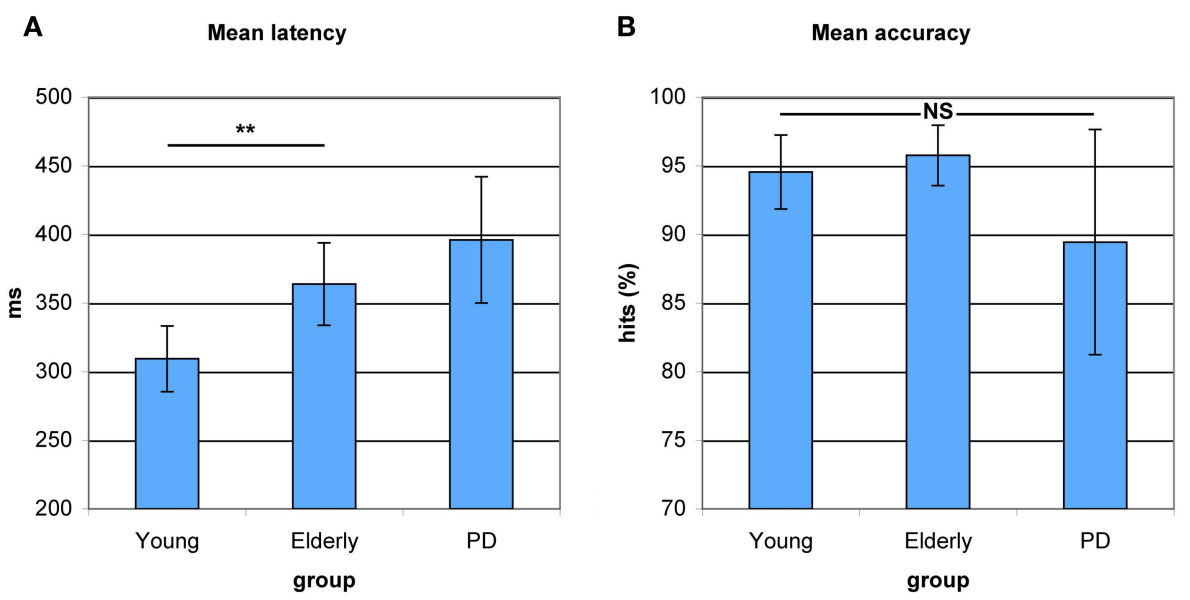

FIGURE 2 |Antisaccade latency and accuracy in young healthy participants, healthy elderly, and Parkinson's patients (PD). (A) Increased average antisaccade onset latency in PD patients as compared to healthy elderly and in healthy elderly as compared to young participants. No significant differences in latency between healthy elderly and PD. (B) No significant differences between groups on average antisaccade accuracy. ${ }^{*} P<0.05 ;{ }^{*} P<0.01$.
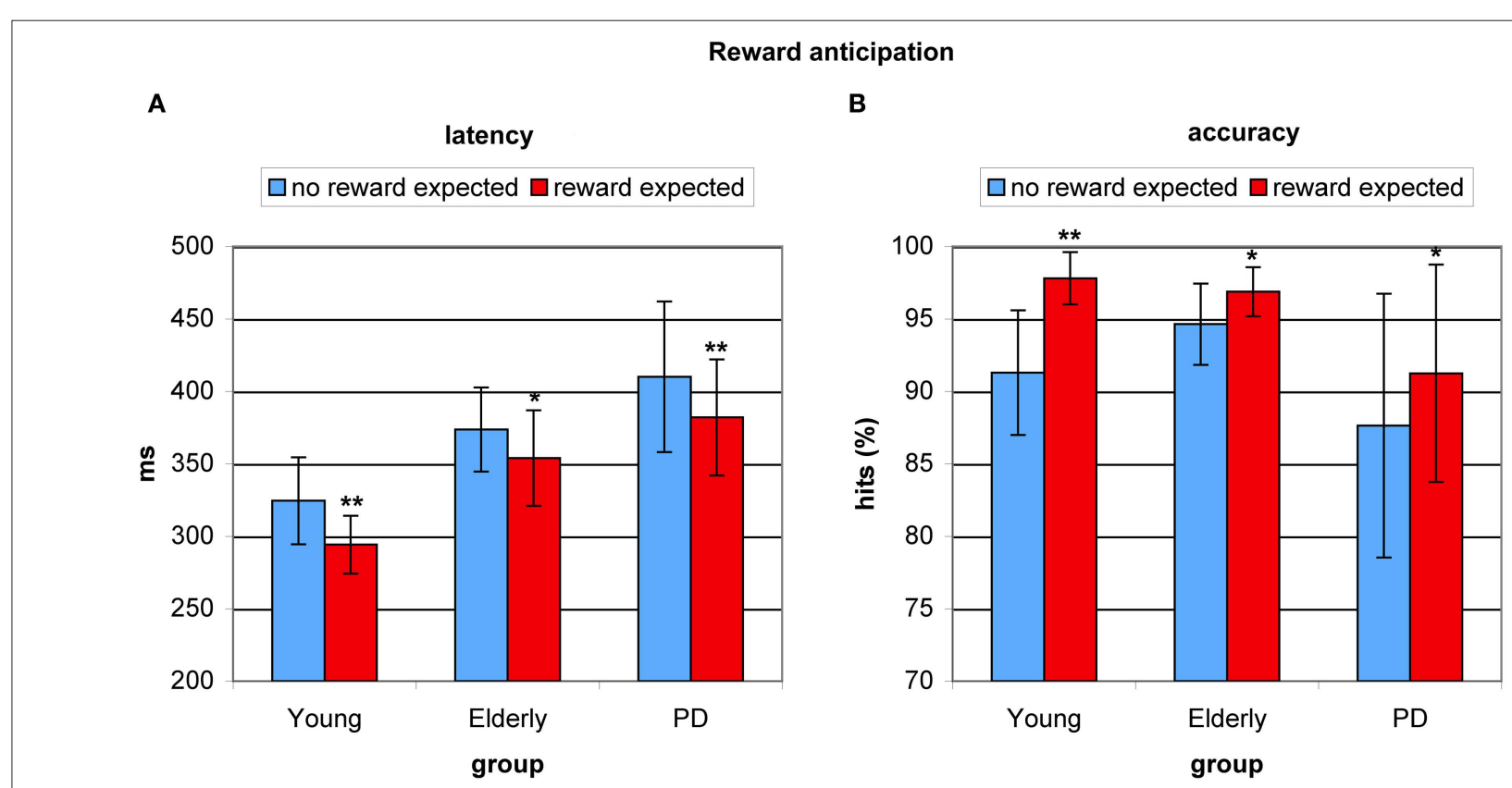

FIGURE 3 | Benefits from reward anticipation in young healthy participants, healthy elderly, and Parkinson's patients (PD). (A) Average antisaccade onset latency was significantly faster on reward anticipation trials (red) than on trials where no reward was expected (blue). (B) Average antisaccade accuracy was significantly higher on reward anticipation trials (red) than when no reward was anticipated (blue). ${ }^{*} P<0.05$; ${ }^{*} P<0.01$.

$(F(1,19)=11.910, p<0.003)$. There were no between-group differences in the effect of specific action preparation $(F(2,53)=0.541$, $p<0.586$, see Figure 4A). Specific preparation also had significant effects on antisaccade accuracy $(F(2,53)=12.882, p<0.001)$ with a spatial preparation benefit of $4.5 \%$ for young adults $(F=10.289$, $p<0.005$ ) and a marginally significant benefit of $2.5 \%$ for healthy elderly $(F=3.518, p<0.079)$ and a benefit of $3.9 \%$ for PD patients $(F=3.330, p<0.085)$, although no significant differences between groups were indicated $(F(2,53)=0.541, p<0.586$, see Figure 4B).

\section{EFFECT OF PROLONGED PREPARATION TIME ON ANTISACCADE LATENCY}

\section{AND ACCURACY}

Prolonged preparation time before appearance of the antisaccade target resulted in significantly faster latencies $(F(2,53)=27.505$, $p<0.0001)$. This cue-target interval benefit amounted $31 \mathrm{~ms}$ in young adults $(F(1,17)=22.947, p<0.0001), 24 \mathrm{~ms}$ in healthy elderly $(F(1,17)=18.759, p<0.001)$, and $33 \mathrm{~ms}$ in PD patients $(F(1,19)=45.55, p<0.0001)$. Again, no difference in the degree of effect was found between groups $(F(2,53)=0.756, p<0.475$, 
see Figure 5A). Longer preparation time had no beneficial effect on accuracy in young participants $(F=0.465, p<0.505)$ or in elderly $(F=2.560, p<0.129)$, but PD patients significantly improved their accuracy $(F=11.604, p<0.003)$, which could be ascribed to patients' low accuracy on trials with shorter cue-target intervals, see Figure 5B.

\section{HIGHER-ORDER INTERACTIONS}

No interaction effects were observed between reward anticipation and specific action preparation, between reward anticipation and cue-target interval, or between specific action preparation and cue-target interval; and these higher-order interactions were not modulated further by Group (all $p>0.2$ ).

\section{Specific action preparation}

A

latency

$\square$ non-specific $\square$ specific

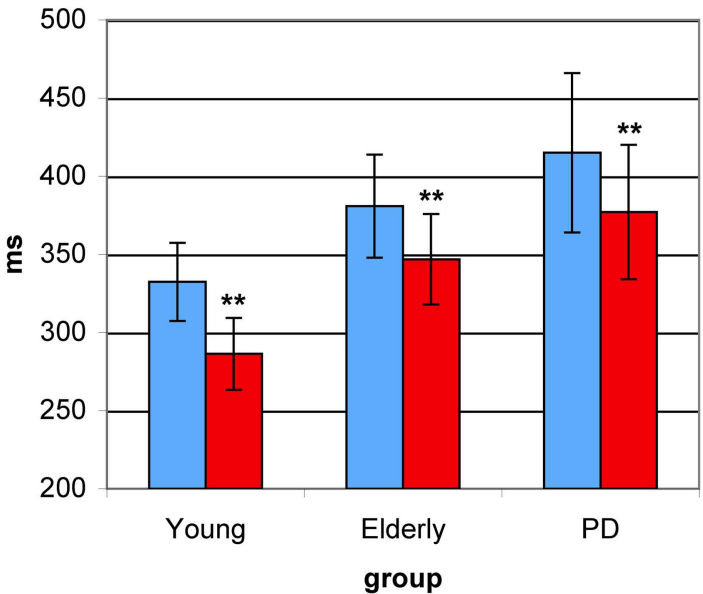

FIGURE 4 | Benefits from specific spatial preparation in young healthy participants, healthy elderly, and Parkinson's patients (PD). (A) Average antisaccade onset latency was significantly faster on specific spatial preparation trials (red) than on trials where a neutral instruction cue was
B

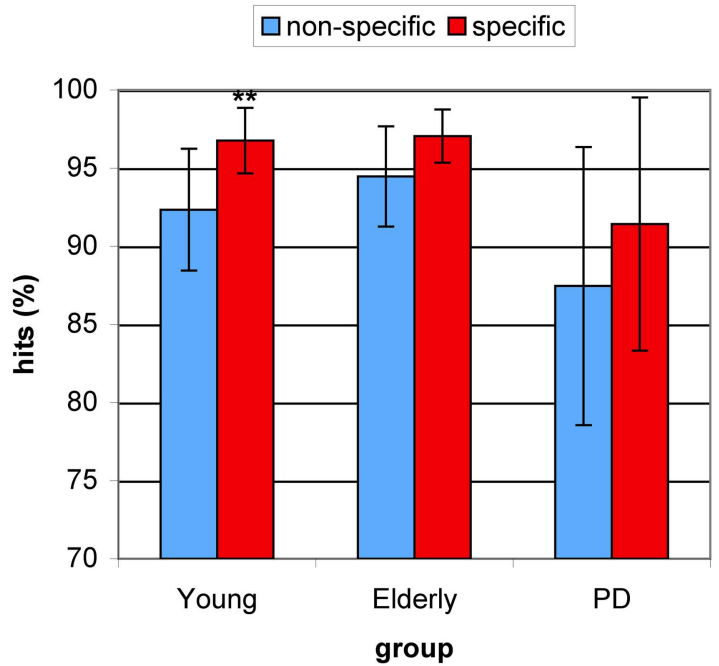

displayed (blue) in all groups. (B) Average antisaccade accuracy was significantly higher on specific preparation trials (red) than on non-specific trials (blue) in the young, and marginally higher in older participants and PD patients. ${ }^{*} P<0.05$; ${ }^{*} P<0.01$.
A

latency

$\square 4750 \mathrm{~ms} \square 5750 \mathrm{~ms}$

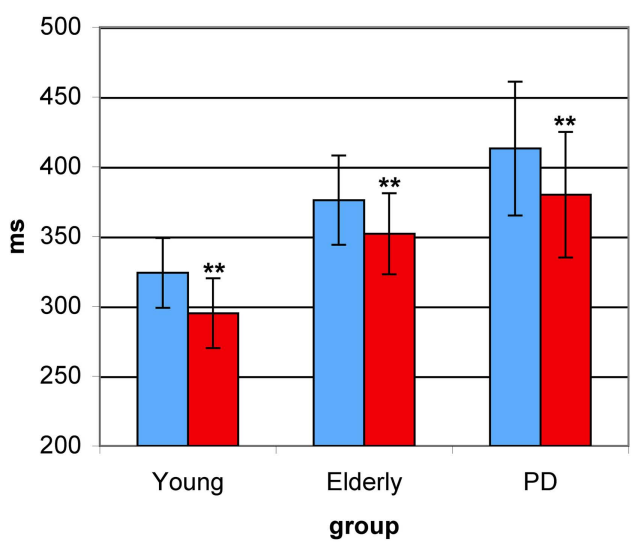

Prolonged preparation time

B

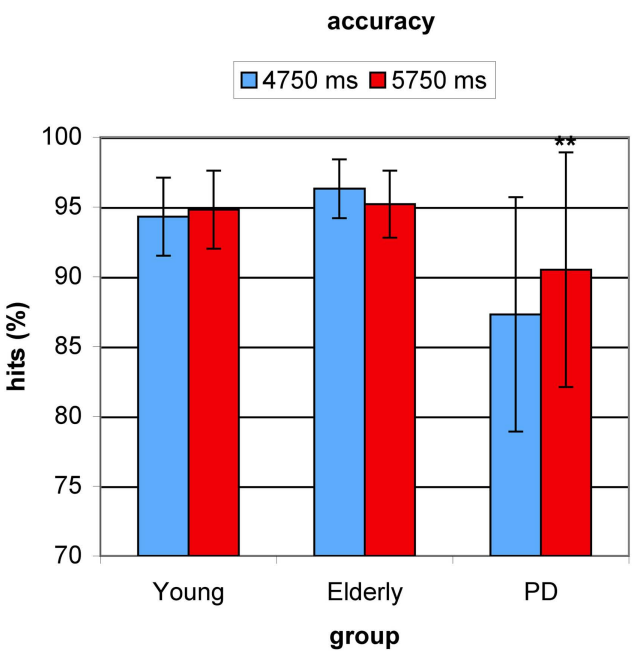

FIGURE 5 | Benefits from prolonged preparation time in young healthy participants, healthy elderly, and Parkinson's patients (PD). (A) Average antisaccade onset latency was significantly faster on prolonged preparation trials (red) than on trials with relatively less time (blue) in all groups. (B) Only in PD patients' average antisaccade accuracy was significantly higher with longer preparation time (red). ${ }^{*} P<0.05 ;{ }^{* *} P<0.01$. 


\section{PREMATURE EYE MOVEMENTS (FIXATION BREAKS)}

Young adults generally exhibited fewer antisaccade trials with fixation breaks $($ mean $=4.2 \pm 9.4 \%)$ than elderly $($ mean $=8.4 \pm 7.4)$ and patients ( mean $=9.0 \pm 13.7 \%$ ), although this trend did not reach significance $(F(1,36)=2.20, p<0.145)$. Reward anticipation did not affect fixation breaks in any of the groups. Adding specific cues led to a significant increase in fixation breaks in elderly (non-specific mean $=4.4 \pm 5.3 \%$; specific mean $=12.4 \pm 10.7 \%(F(1,17)=19.084$, $p<0.0001$ ) and patients (non-specific mean $=7.0 \pm 12.2 \%$; specific mean $=10.9 \pm 16.0 \%(F(1,19)=5.238, p<0.034)$, and a marginal increase in young (non-specific mean $=1.9 \pm 4.7 \%$; specific mean $=6.5 \pm 14.3 \%(F(1,17)=3.841, p<0.068)$. No significant effects of cue-target intervals on fixation breaks were found in any of the groups.

\section{ANTISACCADE PERFORMANCE IN PD PATIENTS WITH MILD MOTORIC SYMPTOMS COMPARED TO PATIENTS WITH SEVERE MOTORIC SYMPTOMS}

To explore associations between antisaccade performance and the severity of motor symptoms, the PD patient group was split into two equal subgroups based on the motoric subscale scores of the Unified Parkinson's Disease Rating Scale (UPDRS). Subjects with scores below 20 were regarded as patients with mild motoric symptoms, while higher scoring patients were regarded as patients with severe motoric symptoms. The two groups did not differ in terms of age $(F(1,17)=2.796, p<0.114)$. When restricting the patient group to severe-symptom patients, the difference in antisaccade latency between older adults and PD patients reached significance $(F(1,26)=6.039, p<0.022)$. Severe-symptom patients had significantly slower antisaccade onset latencies than mild-symptom patients $(F(1,19)=4.956, p<0.041)$. Mild-symptom patients and healthy elderly did not differ in onset latency $(F(1,26)=0.064$, $p<0.802$, see Figure 6A). Patients with severe symptoms furthermore showed significantly lower antisaccade accuracy compared to patients with mild symptoms $(F(1,19)=7.894, p<0.013)$ and elderly $(F(1,26)=12.442, p<0.002$, see Figure 6B). Within the patient group, mild-symptom patients showed a marginally lower number of fixation breaks (mean $=4.7 \pm 5.9 \%$ ) than severe-symptom patients $($ mean $=16.0 \pm 9.2 \%, F(1,19)=3.138, p<0.096)$. These significant differences in antisaccade latency and accuracy and the marginal differences in premature responses (fixation breaks) were not due to age differences. In sum, patients with more severe motoric symptoms as measured by the UPDRS, displayed decreased antisaccade control compared to patients with mild symptoms.

\section{DISCUSSION}

Our results indicate beneficial effects of motivational incentives on cognitive performance and alleviating effects on cognitive decline. These beneficial effects were evident in healthy young and older adults and in patients with Parkinson's disease. Young adults showed improved antisaccade preparation when a reward was at stake; older adults and PD patients could utilize reward prospect to improve their impaired antisaccade preparation. Elderly participants and PD patients were not only better prepared by means of commonly used preparatory aids (i.e., more preparation time and advance information on the upcoming response) but also by means of motivational incentives. These beneficial effects of motivational incentives on cognitive action preparation in healthy and pathological aging further support views that question the notion of monotonic deterioration of cognitive function with age (Allen et al., 2001). They indicate that age-related declines can be modulated by emotion-cognition interactions.

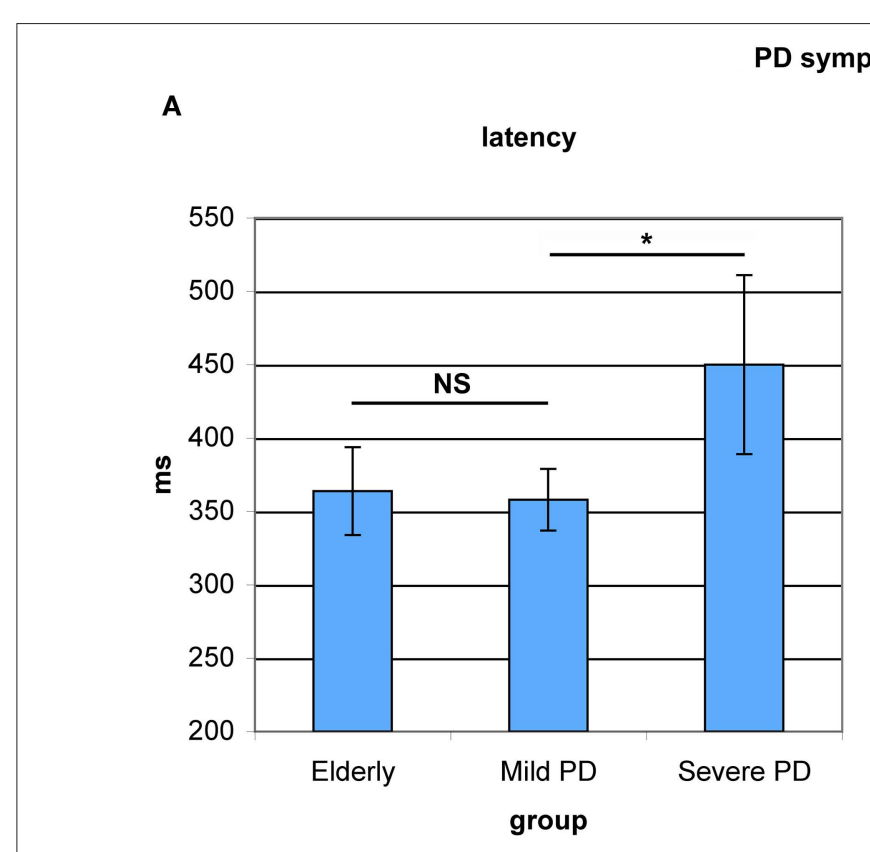

B

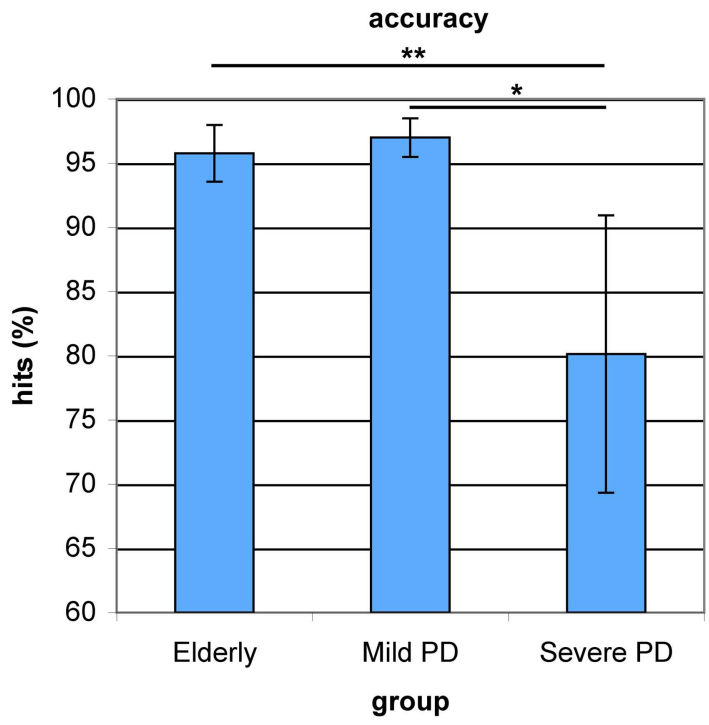

FIGURE 6 | Antisaccade latency and accuracy in healthy elderly and Parkinson's patients with mild and severe motoric symptoms. (A) Mild-symptom patients and healthy elderly did not differ in onset latency. Severe-symptom patients showed significantly slower onset latencies. (B) Mild-symptom patients and healthy elderly did not differ in accuracy. Severe-symptom patients were significantly less accurate. ${ }^{*} P<0.05 ;{ }^{*} P<0.01$. 
In line with the predominant view that cognitive control deteriorates in aging and Parkinson's disease, and in line with previous findings on age-related changes in antisaccade control specifically (Eenshuistra et al., 2004; Chan et al., 2005; Amador et al., 2006; Abel and Douglas, 2007), the current results showed decline in antisaccade preparation in PD patients with severe motor symptoms as compared to healthy seniors. Mild-symptom patients and healthy elderly did not differ in antisaccade preparation. Healthy seniors showed declining antisaccade preparation as compared to young adults. Decline was evident on several oculomotor parameters and most pronounced on antisaccade onset latencies. In line with the notion that in general, elderly tend to prefer accuracy over speed, elderly showed no decreased antisaccade accuracy as compared to young adults. As compared to the young group, elderly and PD patients exhibited slightly, but not significantly more premature eye movements. Premature eye movements, however significantly increased in elderly and PD patients but only slightly in the young, when more specific information on the upcoming movement was available in advance. Presumably, this increase in premature eye movements reflects difficulties with the inhibition of externally guided movement and/or the suppression of strongly activated prepotent action plans. On the one hand, the choice for medicated PD patients represents a limitation of the current study as we cannot exclude differential effects of medication on behavior. On the other hand, PD patients generally take medication in their daily routine, such that the results can be generalized to clinical practice. Exploratory analysis within the $\mathrm{PD}$ patient group revealed that patients with more severe motoric symptoms (as indexed by their motoric subscore on the UPDRS) displayed more signs of antisaccade decline, such as longer antisaccade latencies and lower accuracy compared to mildly affected patients, who initiated saccades equally fast as healthy adults. This suggests that dopaminergic medication was not sufficient to raise the patient's antisaccadic preparation up to the level of the healthy older adults.

One intriguing observation was that despite the different extents of observed antisaccade performance decline in elderly and PD patients, reward prospect led to beneficial effects on antisaccade latency and accuracy in young, elderly, and PD patients alike. Young, elderly and PD patients benefited from reward prospect to the same extent, confirming that the expectancy of reward is a powerful mediator of cognitive control, even if cognitive control suffers from decline. The expectation of rewarding incentives thus seems to support older adults' and $\mathrm{PD}$ patients' ability to anticipate, prepare and initiate goal-directed behavior.

These findings imply that despite a general decline in the action preparation system as indexed by increased antisaccade latency, action preparation processes remain susceptible to the prospect of future reward. It could thus be argued that the neural representations of upcoming rewards influence neural systems concerned with action preparation and oculomotor control. Although the current behavioral results cannot exclude or confirm neural links between reward anticipation and action preparation systems in the brain, they suggest intact cross-talk among subcortical circuits that allow goal-directed action to be modified by motivational factors. The motivational neural reward anticipation signal presumably activates the reward network including the amygdala, orbitofrontal cortex, the more ventral and dorsal striatum (nucleus accumbens, putamen, and caudate), leading to an release of caudate/SNr inhibition on the executive oculomotor structure superior colliculus. This transiently decreased inhibitory force on superior colliculus may improve goaldirected oculomotor control. Thus reward anticipation might compensate for the age- and disease-related dopaminergic loss in the SNc projections to the caudate/SNr and may thus play a compensatory role in "boosting" the efficiency of interactions between motivational and impaired cognitive control processes.

However the reward-related modulation of the oculomotor control system may not only apply to the basal ganglia part of the oculomotor network. Although speculative, it could extend to the cortical eyefields (frontal, supplementary, and parietal eyefields) and to parts of the visual cortex. Human research shows that more preparatory activation in the frontal eyefields leads to a faster antisaccade latency (Connolly et al., 2002, 2005). The compensatory effect on the antisaccade latency, therefore, could theoretically stem from a propagation of reward anticipation signal in the caudate to the frontal eyefields. Complex structural connections enable the striatum to exert a controlling influence over the cerebral cortex via a series of reentrant corticobasal ganglia circuits. Thus reward anticipation may increase the level of preparation in the intact cortical eyefields and thereby compensate for age- and disease-related subcortical decrements in the oculomotor network.

Taken together, it seems plausible that motivational reward processes in the caudate enhance preparatory activation in subcortical as well as in cortical nodes of the oculomotor network. Several neurotransmission mechanisms may underlie the remedial effect of positive incentives on declining antisaccadic control. Given the convergence of dopamine and glutamate inputs at the spiny projection neurons in the striatum (Burns et al., 1994), an interaction between dopamine and glutamate might underlie striatal processes of reward anticipation and action preparation. However, it cannot be fully excluded that alterations in other neurotransmitter systems may also have an effect on declining oculomotor control and on remediating incentive effects. Non-dopaminergic changes such as noradrenergic, serotonergic, and cholinergic deafferentation of the cortex also occur in PD and can inflict cognitive deficits (Agid et al., 1987). Evidence from non-human primates suggests that basal ganglia dopamine acts to speed motor responding in response to rewarding cues (Niv, 2007; Niv et al., 2007). Among at least five types of DA receptors, mainly D1 and D2 receptors are expressed in caudate projection neurons (Nakamura and Hikosaka, 2006). Tendencies to speed responses to obtain large rewards have been found to be dependent on striatal D1 receptors (Dalley et al., 2005; Berridge, 2007). In contrast to this D1 modulated speeding in reward-motor tasks, evidence from saccade tasks in non-human primates suggests D2 receptor modulation of reward dependent improvements of performance (Nakamura and Hikosaka, 2006). Injections of $\mathrm{a}$ D1 antagonist and a D2 antagonist into the region of the caudate where saccade-related neurons are clustered while the monkey performed a reward-biased saccade task showed that D1 antagonists attenuate the reward modulation of saccade behavior whereas D2 antagonists into the same region enhanced the rewarddependent changes.

Future studies of Parkinson patients on versus off medication, could provide some information on the role of dopaminergic mechanisms in the remedial effects on antisaccadic performance. 
A recent study of reward learning in Parkinson patients on/off dopaminergic medication showed that patients adapted their behavior to maximize expected reward value dependent on their medication status (Moustafa et al., 2008). Whilst off dopaminergic medication, patients slowed their responses to avoid low expected values, but succeeded less well in speeding responses when a reward was at stake. The opposite pattern emerged when the same patients were on medication; patients showed better response speeding, and worse response slowing, to maximize expected value.

The current behavioral findings are in line with neuroimaging research showing intact neural representations of future reward in the aging striatum (Samanez-Larkin et al., 2007). The observation however that older adults and PD patients can use this intact neural representation of reward anticipation (a positive motivational incentive) to optimize their preparatory processes, cannot necessarily be generalized to cognitive contexts with negative motivational incentives. Samanez-Larkin et al.(2010) recently showed that older adults were not able to optimally use their intact neural reward anticipation system in risky financial decision making. Elderly made more suboptimal choices when they were to choose risky assets. The authors deemed it likely that these decrements in value-based decision making are explained by the fact that value-based decision making requires the weighting of behavioral alternatives, that may lead to either gain or loss. During anticipation of rewards older adults show similar neural responses as young adults but they show reduced neural activation during the anticipation of losses (Samanez-Larkin et al., 2007). The latter, together with the observation that older adults tend to shift their attention away from negative stimuli and attend to positive information instead (Mather and Carstensen, 2003, 2005), may have led to the underestimation of loss and therefore to more risky and suboptimal decisions.

In this respect, one limitation of the current task is that it built solely on reward prospect and not on the prospect of loss, such that the question of how the anticipation of loss influences action preparation was not tested. The impact of negative incentives might be an intriguing question for future research, as due to the decreased activation of the neural loss anticipation system (Samanez-Larkin et al., 2007) together with the observed differential attentional bias for negative and positive information (Mather and Carstensen, 2003), it seems likely that negative motivational incentives, i.e., the prospect of loss, might have a different impact on action preparation in older adults than in the young. Future approaches to the effect of loss anticipation on declining antisaccade control may ideally contain a sequence of experiments, starting with a separate (punishment only) approach before moving to combined reward-punishment designs. This might be necessary as previous evidence (from healthy young adults) suggests that the reward effect on oculomotor control disappears in the presence of punishment trials. The authors deemed it likely that the participants increased caution to all incentive (reward and punishment) trials (Blaukopf and DiGirolamo, 2006).

As of yet, age-related changes in neural reward anticipation have mainly been studied in isolation. Recently however, the interaction of neural reward processing with other cognitive functions and their underlying neural systems, such as the action control system, has gained increasing interest (Haber and Knutson, 2010). Initial evidence suggests coordinated activity between distant brain regions responsible for reward processing (Cohen et al., 2009). The current results encourage further neural network investigations on the role of coordinated brain network activity in motivated behavior. Behavioral benefits from motivation might be associated with changes in functional brain connectivity on a larger scale, e.g., between frontostriatal reward and action preparation structures.

Another important factor that emerges from the present and previous results, is the importance of the training of the motivational aspects of the instructions that need to be kept in working memory when performing a cognitive task. Samanez-Larkin et al. (2007) found intact neural reward anticipation processes specifically in the absence of learning (after the learning process of reward cue-reward associations was completed). Therefore it might be important for researchers doing experiments with motivation based instructions to keep in mind that, compared to young adults, elderly and PD patients are generally hampered on stimulus-response learning (Shohamy et al., 2004; Marschner et al., 2005). Reward instruction cues, as they were used in the current study require participants to form reward cue-reward associations. Impairments in the learning of associations between the cue signaling reward and the actual reward might conceal intact reward anticipation processes. When the formation of associations is weakened, it could appear as a failure to capitalize on the neural reward anticipation system, whereas it represents an indirect effect of non-available reward associations in working memory when preparing the response. Hence, one might circumvent the effect of potential learning impairments on reward anticipation processes by the training of abstract reward cues (as in the current and in Samanez-Larkin's study) or by using explicit and concrete rewards. Once the learning of reward cues is accomplished the intact reward anticipation processes might become evident.

In sum, the results confirmed that the preparation of goal-directed actions declined in $\mathrm{PD}$ patients compared to healthy seniors, and in healthy seniors compared to young adults. Due perhaps to deterioration in dopaminergic striatal circuitry, antisaccade performance is subject to decline in healthy seniors, and even more in individuals with Parkinson's disease. The prospect of future reward however provided a motivational incentive for optimizing oculomotor preparation in all groups alike. These results suggest that reward-processing networks and oculomotor-control networks in the brain are connected in such a way (presumably with the dorsal striatum as a common nexus) that reward anticipation can facilitate oculomotor control and alleviate the deficiencies experienced by healthy seniors and PD patients. Future investigations should examine whether the observed effects are specific for the current task or generalize to other tasks that show age- and PD-related cognitive decrements (e.g., in task switching) and have been related to dopaminergic denervation in the caudate (e.g., impaired verbal episodic memory, attention, and response inhibition; Rinne et al., 2000; Bruck et al., 2005). Future studies on network processes may help to localize the neural link between reward prospect and action preparation that enables alleviating effects of motivational incentives on cognitive decline. 


\section{REFERENCES}

Abel, L. A., and Douglas, J. (2007). Effects of age on latency and error generation in internally mediated saccades. Neurobiol. Aging 28, 627-637.

Agid, Y., Javoy-Agid, E., and Ruberg, M. (1987). "Biochemistry of neurotransmitters in Parkinson's disease”, in Movement disorders, eds C. D. Marsden and S. Fahn. (Butterworth: London), 166-230.

Allen, P. A., Hall, R. J., Druley, J. A., Smith, A. F., Sanders, R. E., and Murphy, M. D. (2001). How shared are age-related influences on cognitive and noncognitive variables? Psychol. Aging 16, 532-549.

Amador, S. C., Hood, A. J., Schiess, M. C., Izor, R., and Sereno, A. B. (2006). Dissociating cognitive deficits involved in voluntary eye movement dysfunctions in Parkinson's disease patients. Neuropsychologia 44, 1475-1482.

Bäckman, L., and Farde, L. (2001). Dopamine and cognitive functioning: brain imaging findings in Huntington's disease and normal aging. Scand. $J$. Psychol. 42, 287-296.

Backman, L., Nyberg, L., Lindenberger, U., Li, S. C., and Farde, L. (2006). The correlative triad among aging, dopamine, and cognition: current status and future prospects. Neurosci. Biobehav. Rev. 30, 791-807.

Berridge, K. C. (2007). The debate over dopamine's role in reward: the case for incentive salience. Psychopharmacology (Berl.) 191, 391-431.

Blaukopf, C. L., and DiGirolamo, G. J. (2006). Differential effects of reward and punishment on conscious and unconscious eye movements. Exp. Brain Res. 174, 786-792.

Bodi, N., Keri, S., Nagy, H., Moustafa, A., Myers, C. E., Daw, N., Dibo, G., Takats, A., Bereczki, D., and Gluck, M. A. (2009). Reward-learning and the novelty-seeking personality: a between- and within-subjects study of the effects of dopamine agonists on young Parkinson's patients. Brain 132, 2385-2395.

Bonin-Guillaume, S., Possamaï, C.A., Blin, O., and Hasbroucq, T. (2000). Stimulus preprocessing, response selection, and motor adjustment in the elderly: an additive factor analysis. Curr. Psychol. Cogn. 19, 245-255.

Brown, M. R., Vilis, T., and Everling, S. (2007). Frontoparietal activation with preparation for antisaccades. J. Neurophysiol. 98, 1751-1762.

Bruck, A., Aalto, S., Nurmi, E., Bergman, J., and Rinne, J. O. (2005). Cortical 6 -[18F]fluoro-L-dopa uptake and frontal cognitive functions in early Parkinson's disease. Neurobiol. Aging 26, 891-898.
Burns, L. H., Everitt, B. J., Kelley, A. E. and Robbins, T.W. (1994). Glutamatedopamine interactions in the ventral striatum: role in locomotor activity and responding with conditioned reinforcement. Psychopharmacology (Berl.) 115, 516-528.

Butler, K. M., and Zacks, R. T. (2006). Age deficits in the control of prepotent responses: evidence for an inhibitory decline. Psychol. Aging 21, 638-643.

Butler, K. M.,Zacks, R. T., and Henderson, J. M. (1999). Suppression of reflexive saccades in younger and older adults: age comparisons on an antisaccade task. Mem. Cognit. 27, 584-591.

Chan, F., Armstrong, I. T., Pari, G., Riopelle, R. J., and Munoz, D. P. (2005). Deficits in saccadic eye-movement control in Parkinson's disease. Neuropsychologia 43, 784-796.

Cohen, M. X., Schoene-Bake, J. C., Elger, C. E., and Weber, B. (2009). Connectivity-based segregation of the human striatum predicts personality characteristics. Nat. Neurosci. 12 , 32-34.

Connolly, J. D., Goodale, M. A., Goltz, H. C., and Munoz, D. P. (2005). fMRI activation in the human frontal eye field is correlated with saccadic reaction time. J. Neurophysiol. 94, 605-611.

Connolly, J. D., Goodale, M. A., Menon, R.S., and Munoz, D. P. (2002). Human fMRI evidence for the neural correlates of preparatory set. Nat. Neurosci. 5, 1345-1352.

Cromwell, H. C., and Schultz, W. (2003). Effects of expectations for different reward magnitudes on neuronal activity in primate striatum. J. Neurophysiol. 89, 2823-2838.

Cropley, V. L., Fujita, M., Innis, R. B., and Nathan, P. J. (2006). Molecular imaging of the dopaminergic system and its association with human cognitive function. Biol. Psychiatry 59, 898-907.

Dalley, J. W., Laane, K., Theobald, D. E., Armstrong, H. C., Corlett, P. R., Chudasama, Y., and Robbins, T. W. (2005). Time-limited modulation of appetitive Pavlovian memory by D1 and NMDA receptors in the nucleus accumbens. Proc. Natl. Acad. Sci. U.S.A. 102, 6189-6194.

Eenshuistra, R. M., Ridderinkhof, K. R., and van der Molen, M. W. (2004). Age-related changes in antisaccade task performance: inhibitory control or working-memory engagement? Brain Cogn. 56, 177-188.

Falkenstein, M., Yordanova, J., and Kolev, V. (2006). Effects of aging on slowing of motor-response generation. Int. J. Psychophysiol. 59, 22-29.

Fearnley, J. M., and Lees, A. J. (1991). Ageing and Parkinson's disease: sub- stantia nigra regional selectivity. Brain 114(Pt 5), 2283-2301.

Fischer, B., Weber, H., and Biscaldi, M. (1993). The time of secondary saccades to primary targets. Exp. Brain Res. 97, 356-360.

Fudge, J. L., Kunishio, K., Walsh, P. Richard, C., and Haber, S. N. (2002) Amygdaloid projections to ventromedial striatal subterritories in the primate. Neuroscience 110, 257-275.

Goerendt, I. K., Lawrence, A. D., and Brooks, D. J. (2004). Reward processing in health and Parkinson's disease: neural organization and reorganization. Cereb. Cortex 14, 73-80.

Grahn, J.A., Parkinson, J.A., and Owen, A M. (2008). The cognitive functions of the caudate nucleus. Prog. Neurobiol. 86, 141-155.

Gunning-Dixon, F. M., Head, D. McQuain, J., Acker, J. D., and Raz, N. (1998).Differential aging of the human striatum: a prospective MR imaging study. AJNR Am. J. Neuroradiol. 19 , 1501-1507.

Haber, S. N., and Knutson, B. (2010). The reward circuit: linking primate anatomy and human imaging. Neuropsychopharmacology, 35, 4-26.

Hallett, P. E. (1978). Primary and secondarysaccades to goals defined by instructions. Vision Res. 18, 1279-1296.

Harrison, M. B., Wylie, S. A., Frysinger, R C., Patrie, J. T., Huss, D. S., Currie, L. J., and Wooten, G. F. (2009). Updrs activity of daily living score as a marker of parkinson's disease progression. Mov Disord. 24, 224-230.

Hikosaka, O., Nakamura, K., and Nakahara, H. (2006). Basal ganglia orient eyes to reward. J. Neurophysiol. 95, 567-584.

Hikosaka, O., Takikawa, Y., and Kawagoe, R. (2000). Role of the basal ganglia in the control of purposive saccadic eye movements. Physiol. Rev. 80 953-978.

Holthoff-Detto, V. A., Kessler, J., Herholz, K., Bonner, H., Pietrzyk, U., Wurker M., Ghaemi, M., Wienhard, K. Wagner, R., and Heiss, W. D. (1997) Functional effects of striatal dysfunction in Parkinson disease. Arch. Neurol 54, 145-150

Hood, A. J., Amador, S. C., Cain, A. E., Briand, K. A., Al-Refai, A. H., Schiess, M. C., and Sereno, A. B. (2007) Levodopa slows prosaccades and improves antisaccades: an eye movement study in Parkinson's disease. J. Neurol. Neurosurg. Psychiatr. 78 565-570.

Hubble, J. P. (1998). Aging and the basal ganglia. Neurol. Clin. 16, 649-657.

Jazbec, S., McClure, E., Hardin, M., Pine, D. S., and Ernst, M. (2005). Cognitive control under contingencies in anx- ious and depressed adolescents: an antisaccade task. Biol. Psychiatry 58, 632-639.

Kaasinen, V., Nagren, K., Hietala, J., Oikonen, V., Vilkman, H., Farde, L., Halldin, C., and Rinne, J. O. (2000). Extrastriatal dopamine D2 and D3 receptors in early and advanced Parkinson's disease. Neurology 54, 1482-1487.

Kawagoe, R., Takikawa, Y., and Hikosaka, O. (1998). Expectation of reward modulates cognitive signals in the basal ganglia. Nat. Neurosci. 1, 411-416.

Kawagoe, R., Takikawa, Y., and Hikosaka, O. (2004). Reward-predicting activity of dopamine and caudate neurons - a possible mechanism of motivational control of saccadic eye movement. J. Neurophysiol. 91, 1013-1024.

Klein, C., Fischer, B., Hartnegg, K., Heiss, W. H., and Roth, M. (2000). Optomotor and neuropsychological performance in old age. Exp. Brain Res. 135, 141-154.

Lauwereyns, J., Watanabe, K., Coe, B., and Hikosaka, O. (2002). A neural correlate of response bias in monkey caudate nucleus. Nature 418, 413-417.

Lewis, S. J., Dove, A., Robbins, T. W., Barker, R. A., and Owen, A. M. (2003). Cognitive impairments in early Parkinson's disease are accompanied by reductions in activity in frontostriatal neural circuitry. J. Neurosci. 23, 6351-6356.

Loveless, N. E., and Sanford, A. J. (1974). Effects of age on the contingent negative variation and preparatory set in a reaction-time task. J. Gerontol, 29, 52-63.

Marklund, P., Larsson, A., Elgh, E., Linder, J., Riklund, K. A., Forsgren, L., and Nyberg, L. (2009). Temporal dynamics of basal ganglia under-recruitment in Parkinson's disease: transient caudate abnormalities during updating of working memory. Brain 132, 336-346.

Marschner, A., Mell, T., Wartenburger, I., Villringer, A., Reischies, F. M., and Heekeren, H. R. (2005). Reward-based decision-making and aging. Brain Res. Bull. 67, 382-390.

Mather, M., and Carstensen, L. L. (2003). Aging and attentional biases for emotional faces. Psychol. Sci. 14, 409-415.

Mather, M., and Carstensen, L. L. (2005). Aging and motivated cognition: the positivity effect in attention and memory. Trends Cogn. Sci. 9, 496-502.

Mell, T., Heekeren, H. R., Marschner, A., Wartenburger, I., Villringer, A., and Reischies, F. M. (2005). Effect of aging on stimulus-reward association learning. Neuropsychologia 43, 554-563.

Mell, T., Wartenburger, I., Marschner, A., Villringer, A., Reischies, F. M., 
and Heekeren, H. R. (2009). Altered function of ventral striatum during reward-based decision making in old age. Front. Hum. Neurosci. 3:34. doi: 10.3389/neuro.09.034.2009.

Milstein, D. M., and Dorris, M. C. (2007). The influence of expected value on saccadic preparation. J. Neurosci. 27, 4810-4818.

Morgante, F., Espay, A. J., Gunraj, C., Lang, A. E., and Chen, R. (2006). Motor cortex plasticity in parkinson's disease and levodopa-induced dyskinesias. Brain, 129, 1059-1069.

Moustafa, A. A., Cohen, M. X., Sherman, S. J., and Frank, M. J. (2008). A role for dopamine in temporal decision making and reward maximization in parkinsonism. J. Neurosci. 28, 12294-12304.

Munoz, D. P., and Everling, S. (2004). Look away: the anti-saccade task and the voluntary control of eye movement. Nat. Rev. Neurosci. 5, 218-228.

Nakamura, K., and Hikosaka, O. (2006). Role of dopamine in the primate caudate nucleus in reward modulation of saccades. J. Neurosci. 26, 5360-5369.

Nieuwenhuis, S., Broerse, A., Nielen, M. M., and de Jong, R. (2004). A goal activation approach to the study of executive function: an application to antisaccade tasks. Brain Cogn. 56, 198-214.

Nieuwenhuis, S., Ridderinkhof, K. R., de Jong, R., Kok, A., and van der Molen, M. W. (2000). Inhibitory inefficiency and failures of intention activation: age-related decline in the control of saccadic eye movements. Psychol. Aging 15, 635-647.

Niv, Y. (2007). Cost, benefit, tonic, phasic: what do response rates tell us about dopamine and motivation? Ann. NY Acad. Sci. 1104, 357-376.

Niv, Y., Daw, N. D., Joel, D., and Dayan, P. (2007). Tonic dopamine: opportunity costs and the control of response vigor. Psychopharmacology (Berl.) 191, 507-520

O’Doherty, J., Dayan, P., Schultz, J., Deichmann, R., Friston, K., and Dolan, R.J. (2004). Dissociable roles of ventral and dorsal striatum in instrumental conditioning. Science 304, 452-454.
Opris, I., and Bruce, C. J. (2005). Neural circuitry of judgment and decision mechanisms. Brain Res. Brain Res. Rev. 48, 509-526.

Prescott, I. A., Dostrovsky, J. O., Moro, E., Hodaie, M., Lozano, A. M., and Hutchison, W. D. (2009). Levodopa enhances synaptic plasticity in the substantia nigra pars reticulata of Parkinson's disease patients. Brain 132, 309-318.

Raemaekers, M., Vink, M., van den Heuvel, M. P., Kahn, R. S., and Ramsey, N. F. (2006). Effects of aging on BOLD fMRI during prosaccades and antisaccades. J. Cogn. Neurosci. 18, 594-603.

Ramnani, N., and Miall, R. C. (2003). Instructed delay activity in the human prefrontal cortex is modulated by monetary reward expectation. Cereb. Cortex 13, 318-327.

Reuter, B., Herzog, E., Endrass, T., and Kathmann, N. (2006). Brain potentials indicate poor preparation for action in schizophrenia. Psychophysiology, 43, 604-611.

Rinne, J. O., Portin, R., Ruottinen, H., Nurmi, E., Bergman, J., Haaparanta, M., and Solin, O. (2000). Cognitive impairment and the brain dopaminergic system in Parkinson disease: [18F]fluorodopa positron emission tomographic study. Arch. Neurol. 57, 470-475.

Roggeveen, A. B., Prime, D. J., and Ward, L. M. (2007). Lateralized readiness potentials reveal motor slowing in the aging brain. J. Gerontol. B. Psychol. Sci. Soc. Sci. 62, P78-P84.

Rosenbaum, D. A. (1980). Human movement initiation: specification of arm, direction, and extent. J. Exp. Psychol. Gen. 109, 444-474.

Samanez-Larkin, G. R., Gibbs, S. E., Khanna, K., Nielsen, L., Carstensen, L. L., and Knutson, B. (2007). Anticipation of monetary gain but not loss in healthy older adults. Nat. Neurosci. 10, 787-791.

Samanez-Larkin, G. R., Kuhnen, C. M., Yoo, D. J., and Knutson, B. (2010). Variability in nucleus accumbens activity mediates age-related suboptimal financial risk taking. J. Neurosci. 30, 1426-1434.
Sato, M., and Hikosaka, O. (2002). Role of primate substantia nigra pars reticulata in reward-oriented saccadic eye movement. J. Neurosci. 22, 2363-2373.

Schmidt, L., d'Arc, B. F., Lafargue, G., Galanaud, D., Czernecki, V., Grabli, D., Schupbach, M., Hartmann, A. Levy, R., Dubois, B., and Pessiglione, M. (2008). Disconnecting force from money: effects of basal ganglia damage on incentive motivation. Brain 131, 1303-1310.

Schott, B. H., Niehaus, L., Wittmann, B. C., Schutze, H., Seidenbecher, C. I., Heinze, H. J., and Duzel, E. (2007) Ageing and early-stage Parkinson's disease affect separable neural mechanisms of mesolimbic reward processing. Brain 130, 2412-2424.

Schultz, W. (2006). Behavioral theories and the neurophysiology of reward Annu. Rev. Psychol. 57, 87-115.

Schultz, W., Apicella, P., Scarnati, E., and Ljungberg, T. (1992). Neuronal activity in monkey ventral striatum related to the expectation of reward. J. Neurosci. $12,4595-4610$.

Selemon, L. D., and Goldman-Rakic, P. S. (1985). Longitudinal topography and interdigitation of corticostriata projections in the rhesus monkey. $J$. Neurosci. 5, 776-794.

Shohamy, D., Myers, C. E., Grossman, S. Sage, J., Gluck, M. A., and Poldrack, R. A. (2004). Cortico-striatal contributions to feedback-based learning. converging data from neuroimaging and neuropsychology. Brain 127 , 851-859.

Smith, G. A., and Brewer, N. (1995) Slowness and age: speed-accuracy mechanisms. Psychol. Aging 10 238-247.

Sterr, A., and Dean, P. (2008). Neura correlates of movement preparation in healthy ageing. Eur. J. Neurosci. 27, 254-260.

Sweeney, J. A., Rosano, C., Berman, R. A. and Luna, B. (2001). Inhibitory contro of attention declines more than working memory during normal aging. Neurobiol. Aging 22, 39-47.

Takikawa, Y., Kawagoe, R., Itoh, H., Nakahara, H., and Hikosaka, O. (2002). Modulation of saccadic eye movements by predicted reward outcome. Exp. Brain Res. 142, 284-291.

Tricomi, E. M., Delgado, M. R., and Fiez, J. A. (2004). Modulation of caudate activity by action contingency. Neuron 41, 281-292.

Tseng, K. Y., Riquelme, L. A., Belforte, J.E., Pazo, J. H., and Murer, M. G. (2000). Substantia nigra pars reticulata units in 6-hydroxydopamine-lesioned rats: responses to striatal D2 dopamine receptor stimulation and subthalamic lesions. Eur. J. Neurosci. 12, 247-256.

Watanabe, K., and Hikosaka, O. (2005). Immediate changes in anticipatory activity of caudate neurons associated with reversal of position-reward contingency. J. Neurophysiol. 94, 1879-1887.

Weiler, J. A., Bellebaum, C., and Daum, I. (2008). Aging affects acquisition and reversal of reward-based associative learning. Learn. Mem. 15, 190-197.

Woodward, T. S., Bub, D. N., and Hunter, M. A. (2002). Task switching deficits associated with Parkinson's disease reflect depleted attentional resources. Neuropsychologia 40, 1948-1955.

Conflict of Interest Statement: The authors declare that the research was conducted in the absence of any commercial or financial relationships that could be construed as a potential conflict of interest.

Received: 01 April 2010; paper pending published: 13 April 2010; accepted: 22 September 2010; published online: 15 October 2010.

Citation: HarsayHA, BuitenwegJIV, Wijnen $J G$, Guerreiro MJS and Ridderinkhof KR (2010) Remedial effects of motivational incentive on declining cognitive control in healthy aging and Parkinson's disease. Front. Ag. Neurosci. 2:144. doi: 10.3389/ fnagi.2010.00144

Copyright (C) 2010 Harsay, Buitenweg, Wijnen, Guerreiro and Ridderinkhof. This is an open-access article subject to an exclusive license agreement between the authors and the Frontiers Research Foundation, which permits unrestricted use, distribution, and reproduction in any medium, provided the original authors and source are credited. 